\title{
Calidad educativa y espacios de trabajo universitarios
}

\section{Quality of education and university workspaces}

Fernández Pérez, R., Gámiz Ruiz, M.A., García Martínez, M.A., Moraga Ávila, J., Peña Sánchez, J., Porras Mínguez, S. (Universidad de Granada)

Fecha de recepción: 28 de mayo de 2012

Fecha de aceptación: 11 de junio de 2012

Localizador: http:www.ugr.es/local/miguelgr/ReiDoCrea-Vol.1-Art.17-Fernandez-Gamiz-Garcia-Moraga-Peña-Porras.pdf

\section{RESUMEN}

La sociedad actual avanza a pasos agigantados, circunstancia que exige de nuestro modelo educativo un cambio paralelo a dichos avances. La educación es uno de los motores de cambio y como tal debe dar respuesta a las exigencias que se le planteen.

Es mucha la bibliografía que podemos encontrar acerca de la calidad educativa, pero muy poca de ésta se centra en la evaluación de la calidad de los espacios educativos como tales.

Desde este estudio se pretende analizar los puntos de vista y exigencias de los estudiantes de la Facultad de Ciencias de la Educación de la Universidad de Granada respecto a dichos espacios educativos.

Palabras clave: Universidad, calidad educativa, espacios educativos y ambiente educativo.

\section{ABSTRACT}

Today's society is moving forward at a rapid pace, a fact that requires that our educational model change in parallel with such developments. Education is one of the drivers of change and as such must respond to the demands made on it.

There is ample literature about the quality of education, but very little of it focuses on assessing the quality of educational spaces as such.

The aim of this study is to examine the views and needs of students at the Faculty of Education at the University of Granada with respect to such educational spaces.

Keywords: University, educational quality, educational spaces and educational environment. 


\section{INTRODUCCIÓN}

En la actualidad, la educación es uno de los motores de desarrollo de la sociedad, por lo que conseguir una educación de calidad a todos los niveles, esto es, desde la educación infantil hasta la universitaria, debe tener una importancia en grado superlativo.

Sin embrago, el concepto de "Calidad" en la educación parece tener una gran diversidad de significados, y no se ha logrado un acuerdo a la hora de definir exactamente cual es su significado, ya que su definición implica un juicio de valor respecto del tipo de educación que se quiere para formar un ideal de persona y de sociedad.

La educación está condicionada por factores dinámicos y cambiantes, por lo que la definición de una educación de calidad también varía en diferentes períodos, de una sociedad a otra y de unos grupos o individuos a otros.

Un parámetro que deberá medir la calidad en la educación será el que se refiere a los espacios en los que se desarrolla dicha formación, teniendo en cuenta que no sólo se refieren a las aulas, sino a todos aquellos lugares que contribuyen al desarrollo integral del individuo en el entorno universitario.

La inmensa mayoría de los estudios tratan de definir y medir los ambientes de aprendizaje de calidad, pero muy pocos se han centrado en la evaluación de los edificios y espacios como tales y las aportaciones de esta evaluación a una formación de calidad y acorde a las exigencias del alumnado como uno de los actores principales del proceso de enseñanza-aprendizaje.

El presente trabajo se centra en el análisis de los espacios existentes en la Facultad de Ciencias de la Educación de la Universidad de Granada, y su adecuación a las demandas del alumnado, con el objetivo de proponer nuevas organizaciones espaciales que contribuyan a crear espacios de estudio y trabajo que respondan a las necesidades planteadas.

\section{MÉTODO Y MATERIALES}

La metodología utilizada para el desarrollo de esta investigación es cualitativa, ya que se ha pretendido la comprensión e interpretación de las respuestas obtenidas a través de su análisis.

El estudio ha versado sobre 6 categorías:

- Accesibilidad al medio físico. Se refiere a la cualidad que tienen los espacios para que cualquier persona pueda llegar a todos los lugares y edificios sin esfuerzos y de forma autónoma.

- Espacios sociales. Áreas internas y externas donde la población universitaria puede reunirse, agruparse o participar en actividades tanto lúdicas como de aprendizaje.

- Espacios docentes. Estancias que tradicionalmente se han dedicado a las actividades de uso estrictamente formativo. Aulas, biblioteca...

- Espacios de servicios. Cafetería, comedor, baños.

- Espacios de circulación. Pasillos. 
- Ergonomía. Estudio del trabajo en relación con el entorno en el que se lleva a cabo.

Para el estudio de estos parámetros se ha confeccionado un cuestionario estructurado en 3 aspectos diferenciados:

Información básica: estudios, sexo, edad, asignaturas por cuatrimestre y horas diarias que los encuestados pasan en la facultad.

Escala de valoración, donde los entrevistados/as muestran su nivel de acuerdo o de desacuerdo respecto a 16 afirmaciones que se han presentado.

Preguntas abiertas, con el objetivo de que los entrevistados/as expresen libremente sus opiniones respecto a los parámetros propuestos.

En este estudio la muestra está compuesta por 40 alumnos/as de la Facultad de Ciencias de la Educación de la Universidad de Granada, seleccionados aleatoriamente, lo que nos ha permitido generalizar los resultados.

\section{RESULTADOS}

Tras el estudio e interpretación de los resultados obtenidos en los distintos parámetros estudiados, podemos destacar:

- Accesibilidad al medio físico.

En general las respuestas de los encuestados/as han valorado positivamente la accesibilidad a la facultad, haciendo una pequeña salvedad en cuanto a las quejas existentes acerca del acceso al aulario, fundamentado en la pérdida de tiempo que conlleva ir de un edificio a otro y por la ausencia de alguna galería que conecte ambos edificios, con las dificultades que esto entraña en épocas de invierno.

- Espacios sociales.

Es en este aspecto, donde el descontento se hace más evidente. La mayoría del alumnado de la facultad, aboga y exige más espacios naturales y de esparcimiento, con sombras, donde se pueda trabajar y disfrutar del tiempo libre al mismo tiempo.

Hacer mención a una demanda que se ha repetido constantemente, la necesidad de que el alumnado disponga de una zona donde poder exhibir y hacer públicos sus trabajos, lo cual permitiría la difusión de ideas, opiniones, además de servir de zona de intercambio cultural.

La idea que más se ha repetido en esta categoría es la falta de emplazamientos destinados a la realización de trabajos en grupo. Como se ha comentado anteriormente, se reclama la necesidad de la existencia de espacios de esparcimiento donde poder trabajar, pero además, es necesario que dentro de los edificios existan zonas donde poder trabajar con los compañeros/as sin tener que molestar al resto de alumnos/as y sin caer en distracciones ambientales, cuestión que trataremos más adelante.

- Espacios docentes.

En este punto debemos hacer una distinción entre las aulas del edificio principal y las del aulario. 
Las aulas del edificio principal ofrecen muchas menos posibilidades que las del aulario, por razones tales como la falta de espacio y la masificación de alumnos/as. Es evidente que cuando se planificó el edificio principal en ningún momento se pensó en que el número de estudiantes desbordaría su capacidad, como consecuencia: aulas muy pequeñas y con escasas posibilidades de distribuciones flexibles del mobiliario, concretamente hechos como el que los pupitres están atornillados al suelo, lo que impide la realización de trabajos grupales o el que un estudiante deba ausentarse momentáneamente del aula, conlleva el que el resto de compañeros/as deban levantarse.

Respecto a las aulas del edificio secundario, las opiniones son diversas, pero en general, el grado de satisfacción respecto a su distribución y tamaño es bastante positivo.

La biblioteca ha sido otro espacio que no ha quedado libre de quejas, principalmente por la falta de bancas donde poder estudiar, sobre todo en épocas de exámenes y siendo la causa de esto, el mal aprovechamiento de los espacios.

- Espacios de servicios.

La cafetería es uno de los espacios mejor valorados, siendo el único inconveniente la falta de espacio, debido a la masificación existente en nuestra facultad. Se ha valorado muy positivamente la terraza, ya que épocas de buena climatología, es un lugar idóneo para descargar tensiones, entablar conversaciones y compartir buenos momentos con los compañeros/as

Respecto al pequeño comedor, las quejas están referidas al tamaño, la inexistencia de luz natural y la aglomeración de alumnos/as a las horas del almuerzo.

- Espacios de circulación.

En nuestra facultad, los pasillos cuenta con una afluencia constante de alumnos, se les ha asignado una función para la que no están construidos, son utilizados como zonas de estudio y, como se refleja de las encuestas, el alumnado piensa que éstos deberían cumplir la función para la que fueron construidos y no servir como zonas de trabajo, ya que es muy fácil perder la atención, desconcentrarse y como consecuencia muchas de las ocasiones en las que se trabaja en ellos una pérdida de tiempo.

- Ergonomía.

Varios aspectos a destacar dentro de esta categoría. El primero de ellos es la ventilación de aire. Es evidente que tras las clases, el ambiente se encuentra demasiado cargado, lo resulta un poco desagradable al incorporarse a las aulas.

Otro aspecto a destacar es la temperatura de las aulas, tanto en invierno como en verano, los encuestados argumentan la necesidad de que deben estar las aulas en condiciones adecuadas para que no sea un factor influyente en el rendimiento académico.

En cuanto a la acústica de las aulas, los componentes de la muestra, se quejan de la pésima calidad en la insonorización de estas, llegando en algunas clases un obstáculo para seguirlas correctamente.

Acerca de los niveles de ruido en la facultad, apuntar que ha sido uno de los aspectos peor valorados por el alumnado, con una mención explícita a las obras que se están llevan a cabo actualmente. 
Por último en lo referente a la iluminación. En las aulas del edifico principal la luz natural es prácticamente inexistente, haciendo un uso abusivo de la luz artificial. En el aulario, los ventanales son mayores, pero por problemas de orientación, la luz natural no es aprovechada convenientemente.

\section{DISCUSIÓN}

Cuando se habla de calidad en la Universidad debemos entender que los espacios físicos también son un factor analizable desde este punto de vista, es decir, no podemos referirnos exclusivamente al profesorado, contenidos, y organización formativa, sino también a los espacios educativos: aulas, biblioteca, lugares de tránsito, espacios de estudio, lugares de reunión públicos, espacios lúdicos, etc.

Concretamente esta Facultad se dedica a la formación de los docentes del mañana, que deberán poner en práctica los conocimientos adquiridos pero también valores, hábitos, y la utilización didáctica de los espacios, en definitiva desarrollar las herramientas necesarias para que sus alumnos sean los ciudadanos del futuro. Con esta premisa queremos recordar que todos los espacios pueden ser educativos y formativos, y que esta Facultad debería ser ejemplo como tal. Dicho esto, hemos constatado en este breve pero premonitorio análisis, que el alumnado se muestra descontento en aspectos que deberían estar configurados correctamente ya que contribuyen de manera significativa a las condiciones que se requieren para mantener la atención y concentración en los momentos en los que se desarrollan la clases, es decir gozar de la ventilación iluminación, y temperaturas adecuadas en las aulas y en el resto de espacios, así como de una acústica correcta que permita seguir las clases de forma adecuada, para lo que se requiere de un estudio que mejore estas condiciones, a fin de lograr que éstos no sean los condicionantes para el rendimiento académico .

En cuanto a la configuración arquitectónica de los dos edificios que se utilizan actualmente para desarrollar los estudios de grado y licenciaturas que se imparten así como los master, entendemos que sería necesaria una reorganización de los espacios con el objetivo de mejorar la movilidad y conexión entre ambos lugares.

Sí entendemos que los espacios son útiles didácticamente y como tal debemos pensarlos, basándonos en una pedagogía abierta, donde se de lugar a la exposición, el debate, la discusión y la puesta en común de actividades enriquecedoras para los estudiantes, sería necesario contar con lugares que permitan la manifestación abierta y pública de los mismos, ya que contribuyen a la participación de todos.

La nuevas formas de trabajo impuestas por las innovaciones educativas, llevadas acabo en la didáctica y desarrollo de la mayoría de las asignaturas que se imparten, implican la necesidad de habilitar zonas de trabajo en equipo que permitan afrontar estas tareas con los requerimientos propios que se exigen para tal objetivo, por tanto sería conveniente reorganizar lugares que faciliten las reuniones en equipos de trabajo con las condiciones optimas para ello.

Los espacios docentes, entendidos como aulas, deben ser versátiles en su configuración, es decir, posibilitar que se puedan reorganizar en función de los contenidos, para lo cual es necesario contar con un mobiliario cómodo y dinámico, que permita reordenar el espacio para facilitar el seguimiento de las clases en un ambiente cómodo y adecuado.

Los nuevos planes formativos, implican que el alumnado pasa muchas horas del día en la Facultad, lo cual hace necesario que lugares como la cafetería o el comedor sean adecuados a las demandas de los usuarios.

130 ReiDoCrea. Revista electrónica de investigación Docencia Creativa. Volumen 1. Páginas 126-131 
La Facultad ofrece una formación integral que debe incluir los espacios como lugares didácticos, se hace necesaria la realización de un estudio pormenorizado que refleje las necesidades de los usuarios de la misma y que al mismo tiempo ofrezca soluciones que permitan una adecuación optima de los recursos y un aprovechamiento, que suponga la satisfacción de todos los que participan en la aventura universitaria.

\section{REFERENCIAS BIBLIOGRÁFICAS}

Andrés Sarasa, J.L. (2000). Intraurbanización: La universidad en el centro de la ciudad. Papeles de Geografía.32, 5-17.

Campos Calvo-Sotelo, P. (2000). La Universidad en España. Historia, Urbanismo y Arquitectura. Madrid: Ministerio de Fomento.

Campos Calvo-Sotelo, P. (2002). El Viaje de la Utopía. Madrid: Editorial Complutense.

Campos Calvo-Sotelo, P. (2009). La educación, un hecho espacial: el "campus didáctico" como arquitectura para el Espacio Europeo de Educación Superior. La Cuestión Universitaria. 5, 99-121.

Campos Calvo-Sotelo, P. (2011). Arquitectura y Universidad en la sociedad contemporánea: innovación abierta y aprendizaje activo en las cuatro escalas espaciales. Cuadernos del Instituto Antonio de Nebrija, 14, 2, 149-182.

Casariego Ramírez, J. y et al. (1989). Universidad y Ciudad: La Construcción del Espacio Universitario. Las Palmas de Gran Canaria: Universidad de Las Palmas de Gran Canaria.

Domenech, J., Viñas, J. (1997). La organización del espacio y del tiempo en el centro educativo. Barcelona: Grao.

Macías, M., García Navarro, J. (2010). Metodología y herramienta verde para la evaluación de la sostenibilidad de los edificios. Informes de la Construcción, vol 62, 517, 87-100. 